\title{
El efecto de consecuencias diferenciales: un caso de investigación traslacional*
}

\section{The Differential Outcomes Effect: a Case of Translational Research}

Recibido: 01 de marzo de 2015| Aceptado: 26 de septiembre de 2015

\author{
Laura Rebeca Mateos Morfín ** \\ CARlos Javier Flores AgUiRre*** \\ Universidad de Guadalajara-IGCAAV, México
}

doi:10.11144/Javeriana.upsy15-2.ecdc

Para citar este artículo: Mateos-Morfín, L.R., \& Flóres-Aguirre, C.J. (2016). El efecto de consecuencias diferenciales: un caso de investigación traslacional. Universitas Psychologica, 15(2), 51-60. http://dx.doi.org/10.11144/Javeriana.upsy15-2.ecdc

* Artículo de investigación. Este trabajo fue apoyado por el proyecto UDG-PTC-1152 otorgado al primer autor por el Programa para el Desarrollo Profesional Docente PRODEP.

** Dra. L. Rebeca Mateos Morfín, Instituto de Gestión del Conocimiento y del Aprendizaje en Ambientes Virtuales (IGCAAV). Correo electrónico: rebeca. mateos@suv.udg.mx

**** Dr. Carlos Javier Flores Aguirre, Centro de Estudios e Investigaciones en Comportamiento (CEIC) Correo electrónico: carlos.flores@cucba.udg.mx

\section{RESUMEN}

La investigación traslacional representa un esfuerzo por integrar los hallazgos derivados de la investigación básica y aproximarlos al ámbito aplicado. El presente trabajo plantea al área de investigación relativa al Efecto de Consecuencias Diferenciales (ECD) como un caso de investigación traslacional caracterizada por su contribución en el aprendizaje de discriminaciones condicionales. Se realiza una revisión que parte de algunos de los trabajos germinales con animales de laboratorio y que continúa con los primeros estudios con humanos. Posteriormente, se revisan algunos estudios que destacan el potencial de aplicación de los Procedimientos con Consecuencias Diferenciales (PCD) como herramienta para el tratamiento de personas con diversos padecimientos asociados a deficiencias en el aprendizaje y la memoria, así como su empleo en el ámbito educativo en situaciones que implican el aprendizaje de discriminaciones condicionales.

Palabras clave

investigación traslacional; discriminación condicional; Efecto de Consecuencias Diferenciales; aprendizaje; memoria

\section{A B S T R A C T}

Translational research represents an effort to integrate the results derived from basic research in order to approach them to the applied field. This paper sets out the area of research in regards to the Differential Outcomes Effect (DOE) as a case of translational research, characterized by its contribution to conditional discrimination learning. A revision starting from several germinal works with laboratory animals and that moves into the first studies with humans. Afterwards, some studies that highlight the potential of the application of the Differential Outcomes Procedures (DOP) are revised as a tool for the treatment of persons with several ailments related to learning and memory deficits, as well as their application to the educational field under situations that involve conditional discrimination learning. Keywords translational research; conditional discrimination; Differential Outcomes Effect; learning; memory 
En el ámbito de la psicología parece ser una práctica común que los tratamientos utilizados en los diversos campos de la disciplina no se conecten y deriven de la investigación básica realizada en los laboratorios. Algunos autores han señalado que esta es una práctica contraria a lo que tradicionalmente sucede en el área médica, en donde se desarrollan una serie de pruebas e investigaciones que permiten llevar los hallazgos de la investigación básica a su aplicación con humanos (Flores, 2011; Laborda, Miguez, Polack, \& Miller, 2012; Mustaca, 2004, 2011; Overmier, 2001, 2007). Este tipo de investigación se reconoce como investigación puente o traslacional, la cual representa un esfuerzo por identificar potenciales aplicaciones de los hallazgos de la investigación básica, ya sea para la innovación de tratamientos o técnicas ya existentes o para la generación de nuevas estrategias de intervención (e.g., Breckler, 2006; Critchfield, 2011; Escobar, 2011, 2012; Lerman, 2003; Mace \& Critchfield, 2010; Ribes, 2009; Santoyo, 2012; Tashiro \& Mortensen, 2006).

Un caso de investigación traslacional se puede reconocer en el llamado Efecto de Consecuencias Diferenciales (ECD), caracterizado por un rápido aprendizaje y un efecto favorable en los niveles terminales de ejecución en tareas que implican discriminaciones condicionales. El Procedimiento de Consecuencias Diferenciales (PCD) consiste básicamente en reforzar con consecuencias específicas las respuestas que se emiten ante estímulos diferentes, por lo que la respuesta que se emite ante cada estímulo se encuentra relacionada a una consecuencia única o particular (Holden \& Overmier, 2014).

El presente trabajo tiene como propósito destacar el potencial de aplicación del PCD mediante la revisión de estudios que muestran el ECD desde la investigación con animales de laboratorio y su extensión gradual con humanos que presentan diversos padecimientos. Si bien el presente documento no es formalmente una revisión sistemática (Petticrew \& Roberts, 2006; Urrútia \& Bonfill, 2010), si ofrece un panorama general que pretende contribuir a la recuperación de hallazgos representativos que hacen evidentes las distintas aplicaciones potenciales del PCD.

\section{Origen y explicación del ECD}

El estudio del ECD surge en la década del setenta en el ámbito de la investigación básica con animales (Trapold, 1970), desde ese entonces, la evidencia acumulada ha dejado ver la replicabilidad del efecto y su generalidad entre especies. En este sentido, es posible encontrar una serie de trabajos en los cuales se muestra este continuo, desde investigaciones con sujetos animales (e.g., Flores \& Mateos, 2010; Mateos, Cabrera, García-Leal, \& Flores, 2011; para una revisión más amplia ver Goeters, Blakely, \& Poling, 1992; Urcuioli, 2005), hasta indagaciones con humanos (e.g., Esteban, Plaza, López-Crespo, Vivas, \& Estévez, 2014; Joseph, Overmier, \& Thompson, 1997; Estévez et al., 2007).

Trapold (1970) postuló que si un organismo es expuesto a una situación en la que en presencia de un estímulo (E1) una respuesta específica (R1) es seguida por una consecuencia particular (C1) y ante un segundo estímulo (E2), otra respuesta (R2) es seguida por otra consecuencia específica (C2), cada uno de los estímulos genera respuestas anticipatorias o de expectativa determinadas por la consecuencia con la que está correlacionado. Adicionalmente, hipotetizó que estas respuestas de expectativa podrían favorecer el control desarrollado por los estímulos, contribuyendo así, a una mayor velocidad de aprendizaje.

Para evaluar esta hipótesis, Trapold expuso a tres grupos de ratas a una tarea de discriminación en la que para un grupo (Grupo E) las respuestas que se emitían en la palanca derecha en presencia de un tono eran seguidas de comida, mientras que las respuestas que se emitían en la palanca izquierda en presencia de un "clicker" eran seguidas de agua azucarada. En otro de los grupos (Grupo C) las respuestas en la palanca derecha en presencia del tono y las respuestas a la palanca izquierda en presencia del "clicker" fueron seguidas por comida; un tercer grupo (Grupo A) fue idéntico al Grupo C excepto que las respuestas correctas (tono-derecha y clicker-izquierda) fueron seguidas únicamente por agua azucarada.

Los resultados mostraron que los sujetos con reforzamiento diferencial (Grupo E) tuvieron por- 
centajes de respuestas correctas más elevados en un menor número de ensayos que el resto de los grupos. Con base en los resultados del experimento anterior, Trapold (1970, Experimento 2) se preguntó si la facilitación en el aprendizaje se debía a una asociación entre estímulos. Empleando un procedimiento pavloviano presentó durante una primera fase un tono seguido por agua y el sonido de un clicker seguido por comida (grupo experimental), mientras que en otro grupo (control) ambos estímulos (tono y clicker) fueron seguidos tanto por agua como por la comida con una base aleatoria $(50 \%$ de los ensayos eran seguidos por comida, mientras que el otro 50\% de ensayos eran seguidos por agua). En una segunda fase, probó si la presentación del tono y el clicker facilitaban el aprendizaje de elección entre dos palancas. Sus resultados mostraron que los sujetos del grupo experimental requirieron menos ensayos que los sujetos del grupo control en aprender a responder en la palanca 1 (R1) en presencia del tono y a responder en la palanca 2 (R2) en presencia del clicker.

Estos resultados llevaron a Trapold a confirmar la hipótesis de que los sujetos desarrollan diferentes expectativas de reforzamiento y que estas pueden contribuir en la precisión del responder, facilitando la velocidad de adquisición en este tipo de tareas, a este efecto de facilitación lo denominó ECD.

Las teorías que se han propuesto para explicar el ECD señalan que es el resultado de interacciones entre contingencias respuesta-estímulo (R-E) y contingencias estímulo-estímulo (E-E), sugiriendo que las expectativas o respuestas anticipatorias son el resultado de una relación de contingencia E-E. De acuerdo con esto, si un estímulo (E1) está consistentemente correlacionado con una consecuencia específica (C1), entonces el primer estímulo (E1) desencadena una respuesta de expectativa, la cual se ha conceptuado como respuesta anticipatoria que simultáneamente desarrolla propiedades de estímulo que favorece el aprendizaje de discriminación (Flores et al., 2005; Goeters et al., 1992; Trapold \& Overmier, 1972; Urcuioli, 2005).

Uno de los estudios que amplió los resultados de Trapold (1970) fue reportado por Peterson, Wheeler y Armstrong (1978), en el cual, utilizan- do palomas como sujetos, reforzaron con comida las respuestas de picoteo ante un estímulo (E1) y con agua las respuestas ante otro estímulo (E2) (Grupo consistente). En otro grupo de sujetos las respuestas ante E1 o E2 fueron seguidas de manera indistinta por agua o comida (Grupo inconsistente). Los principales resultados fueron que los sujetos del Grupo consistente tuvieron un mayor porcentaje de respuestas correctas en menos sesiones que los sujetos del Grupo inconsistente.

Otro hallazgo fue reportado por Alling, Nickel y Poling (1991) quienes administraron un agente amnésico a dos grupos de palomas. Reportaron que las palomas entrenadas bajo un PCD obtuvieron un mayor porcentaje de respuestas correctas en contraste con el otro grupo bajo la condición de consecuencias no diferenciales (PCND). Estos hallazgos son consistentes con los reportados en otros trabajos bajo condiciones similares (e.g., Chatlosh \& Wasserman 1992; Peterson, Wheeler, \& Trapold, 1980; Savage \& Langlais, 1995; Savage, Stanchfield, \& Overmier, 1994).

Como se mencionó anteriormente, la investigación sobre el ECD se gestó en la investigación básica con animales y fue con base en estos hallazgos que se trasladó a la investigación básica con humanos. Un estudio en el que se reportó la generalidad del ECD con seres humanos fue el realizado por Maki, Overmier, Delos y Gutman (1995) quienes entrenaron a dos grupos de niños en un procedimiento de igualación a la muestra. Para un grupo de niños (Grupo experimental), las consecuencias programadas para las respuestas correctas fueron comida y una consecuencia o retroalimentación verbal ("eso está muy bien"); mientras que para el otro grupo (Grupo control) las consecuencias fueron las mismas pero aleatorizadas en el total de los ensayos, es decir, en el $50 \%$ de los ensayos se dio comida a los niños y en el otro 50\% se presentó la consecuencia verbal. Los resultados mostraron que los niños del grupo experimental aprendieron la tarea en un menor número de ensayos y que el porcentaje de respuestas correctas fue más alto en comparación con los niños del grupo control, por lo cual, los autores concluyeron que emplear consecuencias diferenciales facilitó el aprendizaje de la tarea de discriminación. 
Los estudios conducidos en el transcurso de las primeras tres décadas sobre el ECD dieron la pauta para la exploración y generalización de este efecto bajo diferentes condiciones y sujetos en los años posteriores. Como se puede apreciar, el ECD ha mostrado replicabilidad bajo diferentes tareas y generalidad entre especies (Goeters et al., 1992; Urcuioli, 2005), con lo cual se ha podido identificar su potencial de aplicación al tener un fuerte impacto sobre el aprendizaje de discriminación. Dado lo anterior, resulta necesario seguir extendiendo su análisis y potencialidad al caso de trastornos del aprendizaje y la memoria en seres humanos, contribuyendo a reconocer la utilidad del PCD como una herramienta metodológica que puede contribuir al desarrollo de estrategias de intervención enfocadas en el tratamiento de diversos padecimientos.

\section{La contribución del PCD en personas con déficits de aprendizaje}

La evidencia muestra que el uso del PCD contribuye al aprendizaje en poblaciones con algún tipo de déficit o alteración en el desarrollo (Estévez, Fuentes, Overmier, \& González, 2003; López-Crespo, Plaza, Fuentes, \& Estévez, 2009; Malanga \& Poling, 1992). En estos casos, el empleo del PCD ha mostrado ser muy eficaz favoreciendo el aprendizaje de discriminaciones condicionales en niños autistas (Litt \& Schreibman, 1981), niños con retraso mental severo (Saunders \& Sailor 1979), personas con daño cerebral vinculado con aspectos de memoria y aprendizaje (Estévez, Fuentes, Mari-Beffa, González, \& Álvarez, 2000).

Un ejemplo de dichos estudios es el reportado por Joseph, Overmier y Thompson (1997) en el que evaluaron si el empleo de consecuencias diferenciales favorecía la formación de clases de estímulos equivalentes en participantes con síndrome de Prader-Willi.

El síndrome de Prader-Willi es un desorden genético, específicamente, una supresión del cromosoma 15. Las personas que lo presentan tienen dificultades de aprendizaje y retraso mental, además de una baja estatura, problemas de comportamiento y desórdenes de los patrones de alimentación (Hochhalter \& Joseph, 2001).

Los resultados del estudio de Joseph et al. (1997) confirmaron el potencial que tiene el PCD para el aprendizaje de discriminaciones condicionales y en la formación de clases de estímulos equivalentes, toda vez que en los participantes en los que se utilizó el PCD aprendieron mucho más rápido las series de discriminación condicional que aquellos en los que se empleó el PCND. Adicionalmente, reportaron que en los participantes entrenados con consecuencias diferenciales se identificó la formación de clases de estímulos equivalentes (i.e., entre $80 \%$ y $85 \%$ de respuestas correctas), mientras que en los participantes entrenados con consecuencias no diferenciales se observaron porcentajes más bajos (entre $40 \%$ y $60 \%$ de respuestas correctas).

Otro de los trastornos cromosomáticos en los que el uso del PCD ha mostrado su contribución en el aprendizaje, es el síndrome de Down (Estévez, et al., 2003). Dado que se ha reconocido que las personas con síndrome de Down presentan dificultades asociadas a la memoria de trabajo visoespacial, Esteban, et al. (2014), evaluaron la contribución del uso del PCD en el reconocimiento de rostros. Esteban et al. (2014) (Experimento 2) compararon la ejecución de niños con desarrollo típico o normal en relación con adultos con síndrome de Down. La tarea consistió en la presentación de rostros masculinos con expresiones neutras. En un primer momento se les mostraba a los participantes la fotografía de un rostro, en un segundo momento y pasados diferentes intervalos de demora $(1,5$, 10 y 15 s) se les presentaba nuevamente el mismo rostro acompañado de otros. El participante debía seleccionar aquella fotografía que se correspondía al rostro mostrado en el primer momento. Para la conformación de los grupos, se aplicaron pruebas que permitieron igualar el grado de desarrollo de los niños con el de los adultos. De manera general, reportaron que todos los participantes mostraron un decremento en el porcentaje de respuestas correctas conforme se incrementó el intervalo de demora. Sin embargo, el decremento fue mucho más pronunciado tanto en los niños como en los adultos que no fueron entrenados con el PCD. 
El efecto favorable del uso del PCD se ha replicado recientemente en niños nacidos prematuramente, que dada su condición presentan deficiencias en el aprendizaje y en la memoria a corto plazo. Martínez et al. (2012), realizaron dos experimentos para evaluar la contribución del PCD sobre el aprendizaje y la memoria de reconocimiento. En un primer estudio participaron 28 niños, de los cuales 14 eran niños nacidos a las 32 semanas de gestación (prematuros) y los 14 restantes, nacidos entre las semana 37 a 42 (nacidos a término). Se conformaron cuatro grupos, 2 de ellos de niños prematuros, uno entrenado en un PCD y otro entrenado en un PCND. Los 14 niños restantes conformaron dos grupos control (niños nacidos a término), un grupo diferencial y uno no diferencial. Los autores reportaron que los niños entrenados con consecuencias diferenciales, tanto prematuros como llegados a término obtuvieron un mayor porcentaje de respuestas correctas que los grupos de niños entrenados en el PCND.

En un segundo experimento, Martínez et al. (2012) obtuvieron resultados similares al explorar si el uso de un PCD favorecía el reconocimiento visoespacial en niños prematuros, observando que los niños bajo un PCD lograron aprender la tarea y obtuvieron un porcentaje de respuestas correctas notablemente superior al de los niños entrenados en un PCND.

\section{La contribución del PCD en personas con trastornos de la memoria}

Si bien el empleo de consecuencias diferenciales tiene un impacto sobre el aprendizaje en términos de la velocidad de adquisición y de los niveles terminales de precisión, también se ha documentado que favorece la ejecución en tareas que evalúan aspectos vinculados con memoria. En este sentido, la investigación emanada del área ha arrojado hallazgos que han mostrado minimizar algunos de los efectos adversos de diversos desórdenes sobre la memoria, como la demencia alcohólica, el síndrome de Korsakoff, el Alzheimer o simplemente los efectos de la edad.

Un estudio que deja ver la contribución que tiene un entrenamiento con consecuencias diferen- ciales sobre los efectos de la edad en la memoria, fue el reportado por Savage, Pitkin y Careri (1999) con ratas jóvenes ( 3 meses de edad) y adultas (24 meses de edad), el cual mostró que el uso de un PCD resultó en un menor decremento de las respuestas correctas. Específicamente, encontraron ejecuciones superiores en las ratas adultas y jóvenes entrenadas con el PCD, en contraste con las ratas entrenadas con el PCND.

Uno de los desórdenes y disfunciones vinculados con aspectos mnémicos es el síndrome de Korsakoff, el cual se presenta en pacientes alcohólicos crónicos. El síndrome de Korsakoff está relacionado con una deficiencia de tiamina como consecuencia del abuso en la ingesta de alcohol por un largo periodo de tiempo, lo cual se manifiesta en dificultades para aprender nuevas asociaciones y recordar eventos posteriores al inicio del abuso del alcohol. Algunas investigaciones como la de Savage y Langlais (1995), que han empleado modelos animales para evaluar el síndrome de Korsakoff, han reportado que usar un PCD favorece el aprendizaje y aminora los efectos negativos sobre la memoria. Estos hallazgos ponen en evidencia la necesidad de seguir explorando y extendiendo los efectos de las consecuencias diferenciales en humanos que presentan este trastorno (e.g., Hochhalter \& Joseph, 2001; Hochhalter, Sweeney, Bakke, Holub, \& Overmier 2000; Hochhalter, Sweeney, Savage, Bakke, \& Overmier, 2001; Legge \& Spetch, 2009).

Por ejemplo, Hochhalter et al. (2000) utilizando una tarea similar a la reportada por Esteban et al. (2014), evaluaron el uso de un PCD en pacientes diagnosticados con síndrome de Korsakoff. Identificaron que los pacientes con este síndrome lograron una mayor precisión para el reconocimiento de rostros bajo el PCD que con un PCND y que incluso, su desempeño fue casi similar al mostrado por un grupo control conformado por personas sin dicho síndrome.

Los hallazgos reportados en este conjunto de estudios se ven fortalecidos a la luz de trabajos más recientes que muestran de manera consistente que el uso del PCD minimiza los efectos adversos sobre el aprendizaje y la memoria producto de la edad u otros trastornos (e.g., López- 
Crespo et al., 2009; Plaza, Esteban, Estévez, \& Fuentes, 2013; Plaza, Estévez, López-Crespo, \& Fuentes, 2011).

Por ejemplo, López-Crespo et al. (2009) evaluaron la posibilidad de que el uso de un PCD minimizara los efectos de la edad en adultos mayores sobre su capacidad para recordar y reconocer rostros. Para probar lo anterior, formaron dos grupos de participantes (adultos mayores vs. jóvenes), a su vez cada grupo fue subdividido dependiendo del tipo de entrenamiento usado (PCD y PCND). Encontraron que la ejecución de los adultos mayores bajo el PCD mostró un porcentaje de respuestas correctas cercano al $95 \%$ e incluso se observó que la precisión al responder no se vio afectada durante la fase de reconocimiento de rostros (fase de demora). Asimismo, encontraron que su ejecución fue muy similar a la mostrada por los grupos de adultos jóvenes sometidos tanto al PCD como al PCND.

Las aportaciones de la investigación sobre el ECD en el marco de los desórdenes de la memoria, cobra relevancia en el contexto actual, puesto que en los últimos 50 años se ha incrementado de forma considerable el estudio sobre padecimientos asociados al deterioro cognitivo, en especial al caso del Alzheimer. Muestra de lo anterior ha sido el creciente desarrollo de nuevas drogas, así como el reciente énfasis en el uso de modelos animales para su investigación. Este resurgimiento ha sido consecuencia del incremento de casos de dicho desorden en los últimos años (Leslie, 2011; Plaza, Antúnez, Estévez, López-Crespo, \& Fuentes, 2012). Recientemente, Plaza et al. (2012) reportaron que el uso de un PCD favoreció a pacientes con Alzheimer en el reconocimiento de rostros con mayor precisión y con latencias más cortas que los pacientes entrenados en un PCND.

Otra contribución de los PCD ha sido aminorar los efectos de la privación del sueño sobre la memoria. En este sentido, Martella, Plaza, Estévez, Castillo y Fuentes (2012) reportaron que personas privadas de sueño resolvieron de forma más precisa una tarea de reconocimiento de rostros bajo un PCD en relación con aquellos participantes entrenados con un PCND.

\section{El PCD como herramienta pedagógica}

En los trabajos descritos anteriormente es posible identificar distintas aplicaciones del PCD en personas que presentan trastornos o problemas en el desarrollo, sin embargo, existe un conjunto de trabajos en los cuales se ha extendido su utilidad en participantes que no cuentan con algún tipo de alteración (Estévez et al., 2007; Martínez, Estévez, Fuentes, $\&$ Overmier, 2009; Miller, Waugh, \& Chambers, 2002; Minster, Jones, Ellife, \& Mathukumaraswamy, 2006; Mok \& Overmier, 2007).

Un ejemplo de lo anterior son los potenciales de aplicación pedagógica señalados por Mok, Estévez y Overmier (2010), dichos autores identifican en las situaciones educativas, tareas, ejercicios o problemas que el estudiante debe resolver y que implican el aprendizaje de discriminaciones condicionales. Al respecto, sugieren aplicaciones al ámbito educativo, como el utilizarlo en el aprendizaje del significado de símbolos chinos, la química y la geografía, pues existen en dichas materias situaciones ante las cuales el significado de los símbolos es distinto de acuerdo al contexto, o bien, son tan similares entre sí que es necesario que el estudiante logre hacer una discriminación muy fina de las formas, por lo que el uso de los PCD por parte de los profesores contribuiría al aprendizaje de una forma más rápida en los primeros años escolares. Por ejemplo, Miller et al., (2002) evaluaron la efectividad del PCD en el aprendizaje de símbolos japoneses (Kanji) en estudiantes universitarios e identificaron que los estudiantes a los que se les entrenó en dicho procedimiento resultaron con un mayor porcentaje de respuestas correctas, es decir, tuvieron un aprendizaje más rápido del significado de los símbolos que aquellos estudiantes entrenados en un PCND. Estos resultados fueron replicados posteriormente por Easton, Child y López-Crespo (2011) quienes emplearon un PCD para el aprendizaje de estos símbolos y la formación de categorías.

Otros trabajos como el de Estévez et al. (2007) se han dirigido a desarrollar procedimientos que auxilien en la enseñanza de las matemáticas. Estos autores implementaron una tarea de discriminación en la cual estudiantes universitarios debían identi- 
ficar si el significado de los símbolos matemáticos ">" o " $<$ " expresados en una relación era correcta (e.g., +5.26 > -5.29). Los estudiantes debían seleccionar entre uno de los dos símbolos "> o " $<$ " para indicar si la expresión era correcta. Los investigadores encontraron que aquellos estudiantes que fueron entrenados bajo el PCD obtuvieron un mayor número de respuestas correctas a diferencia de los estudiantes entrenados bajo el PCND.

\section{Comentarios finales}

La investigación traslacional posibilita la integración y exploración de manipulaciones que obedecen a una continuidad entre hallazgos caracterizada por su extensión gradual del laboratorio al ámbito aplicado. El ECD es un ejemplo de lo anterior, desde los primeros estudios se mostró como un efecto robusto sobre el aprendizaje y la memoria, tanto en animales de laboratorio (e.g., Trapold, 1970; Trapold \& Overmier, 1972) como en seres humanos (e.g., Joseph et al., 1997; Maki et al., 1995). También es posible identificar una secuencia u orden particular en el cual se fueron realizando las investigaciones sobre el fenómeno, transitando de investigaciones con organismos no alterados o bajo el efecto de algún fármaco y su posterior exploración bajo condiciones en las cuales se empleaban manipulaciones que inducían dificultad en el aprendizaje o la memoria (e.g., Hochhalter et al., 2001; Savage \& Langlais, 1995; Savage, et al., 1994).

Posteriormente, la robustez del ECD llevó a los investigadores a examinar la efectividad del PCD para mejorar el aprendizaje y la memoria en niños con desarrollo normal (e.g., Maki et al., 1995). Los hallazgos derivaron en el reconocimiento del PCD como una herramienta con potencial uso en la rehabilitación de personas con trastornos en el desarrollo como el retraso mental severo y el autismo. Otros investigadores vieron la posibilidad de extender el uso del PCD al tratamiento de padecimientos ligados al deterioro del aprendizaje y la memoria a consecuencia de la edad, lo que incluía al Alzheimer. En este último caso, es posible reconocer que los investigadores en el área han retomado el trabajo de laboratorio con animales (en particular con ratas), para explorar los efectos del PCD en relación con el envejecimiento y sus efectos sobre la memoria y el aprendizaje (e.g., Savage et al., 1999; Mateos, Madrigal, Flores, \& Overmier, 2016). Los resultados positivos han motivado a los investigadores a replicar los hallazgos con adultos mayores y otros síndromes asociados a déficits en la memoria (e.g., Esteban et al., 2014; López-Crespo et al., 2009; Plaza et al., 2012).

El conjunto de evidencia edificado hasta ahora, ha conducido nuevamente a los investigadores a reflexionar sobre el potencial del PCD en el tratamiento de otros trastornos, en particular sobre los efectos adversos de la privación de sueño sobre la memoria (Martella et al., 2012). De manera paralela a la indagación y extensión del ECD en trastornos diversos, otra clase de estudios (de forma relativamente reciente), se ha enfocado en explorar las aplicaciones del PCD en contextos educativos (e.g., Mok et al., 2010) como la enseñanza de idiomas y matemáticas, mostrando hallazgos robustos en condiciones no clínicas que han puesto la atención en la contribución de este procedimiento para la formación de clases de estímulos equivalentes (e.g., Easton et al. 2011; Joseph et al., 1997; Minster et al., 2006).

Los trabajos dirigidos a estudiar el ECD han constituido un área en la que los investigadores del tema sistemáticamente vuelven la mirada a la investigación básica para trasladar los hallazgos a escenarios aplicados. La presente revisión deja ver esta dinámica bajo la cual los hallazgos encontrados en la investigación con animales se han extendido e integrado gradualmente a aplicaciones tanto en escenarios clínicos como educativos, posibilitando la implementación de tratamientos que han contribuido en la calidad de las intervenciones que realizan los profesionales de la disciplina. Si bien en la psicología no se perciben casos claramente representativos de investigación traslacional, es importante llevar a cabo un trabajo de identificación e integración de las investigaciones desarrolladas en algunas áreas de investigación enmarcadas en el análisis de la conducta, puesto que existen casos como el del ECD con amplio potencial de desarrollo. 


\section{Referencias}

Alling, K., Nickel, M., \& Poling, A. (1991). The effects of differential and nondiferential outcomes on response rates and accuracy under a delayedmatching-to-sample procedure. The Psychological Record, 41, 537-549.

Breckler, S.J. (2006). Psychology is translational science. Monitor on Psychology, 37, 6.

Chatlosh, D.L., \& Wasserman, E. (1992). Memory and expectancy in delayed discrimination procedures. En. I. Gormezano y E.A. Wasserman (Eds.). Learning and Memory. (pp. 61-79). Lawrence Erlbaum Associates.

Critchfield, T.S. (2011). Translational contributions of the experimental analysis of behavior. The Behavior Analyst, 34, 3-17.

Easton, A., Child, S., \& López-Crespo, G. (2011). Differential outcomes aid the formation of categorical relationships between stimuli. Behavioural Brain Research, 222, 270-273.

Escobar, R. (2011). De la vida cotidiana al laboratorio: algunos ejemplos de investigación de traducción. Revista Mexicana de Análisis de la Conducta, 37, $32-50$.

Escobar, R. (2012). Investigación de traducción y análisis conductual aplicado: ¿quién debe preocuparse? Revista Mexicana de Investigación en Psicología, 4, 112-120.

Esteban, L., Plaza, V., López-Crespo, G., Vivas, A.B., \& Estévez, A.F. (2014). Differential outcomes training improves face recognition memory in children and in adults with Down syndrome. Research in Developmental Disabilities, 35, 1384-1392.

Estévez, A.F., Fuentes, L.J., Mari-Beffa, P., González, C., $\&$ Álvarez, D. (2000). The differential outcome effect as a useful tool to improve conditional discrimination learning in children. Learning and Motivation, 32, 48-64.

Estévez, A.F., Fuentes, L., Overmier, J.B., \& González, C. (2003). Differential outcome effect in children and adults with Down syndrome. American Journal on Mental Retardation, 108, 108-116.

Estévez, A.F., Vivas, A.B., Alonso, D., Mari-Beffa, P., Fuentes, L.J., \& Overmier, J.B. (2007). Enhancing challenged students recognition of mathematical relations through differential outcomes training. The Quarterly Journal of Experimental Psychology, 60, 571-580.

Flores, C. (2011). De los modelos animales a la práctica psicológica: el surgimiento de algunas técnicas aplicadas a problemas de salud. Suma Psicológica, 18, 115-123.

Flores, C., \& Mateos, L.R. (2010). Probabilidad de reforzamiento diferencial y no diferencial en una tarea de discriminación condicional. Universitas Psychologica, 9, 485-493.

Flores, C., Ortega, D., Reyes, K., Mateos, R., Villanueva, S., \& Amaya, A. (2005). Reversiones parciales y totales muestra-comparativo en igualación a la muestra con consecuencias diferenciales y no diferenciales. Universitas Psychologica, 4, 43-47.

Goeters, S., Blakely, E., \& Poling, A. (1992). The differential outcomes effect. The Psychological Record, 42, 389-411.

Hochhalter, A.K., \& Joseph, B. (2001). Differential outcomes training facilitates memory in people with Korsakoff and Prader-Willi Syndromes. Integrative Physiological and Behavioral Science, 36, 196-204.

Hochhalter, A.K., Sweeney, W.A., Bakke, B. L., Holub, R. J., \& Overmier, J.B. (2000). Improving face recognition in alcohol dementia. Clinical Gerontologist, 22, 3-18.

Hochhalter, A.K, Sweeney, W. A., Savage, L.M., Bakke, B.L., \& Overmier, J.B. (2001). Using animal models to address the memory deficits of Wernicke-Korsakoff syndrome. En M. E. Carroll and J.B. Overmier (Eds.). Animal Research and human health. pp. 281-292. American Psychological Association: Washington.

Holden, J.M., \& Overmier, J.B. (2014). Performance under differential outcomes: contributions of reward-specific expectancies. Learning and Motivation, 45, 1-14.

Joseph, B., Overmier, J.B. \& Thompson, T.I (1997). Food and Nonfood Related Differential Outcomes in Equivalence Learning by Adults with Prader-Willi Syndrome. American Journal of Mental Retardation, 4, 374-386.

Laborda, M. A., Miguez, G., Polack, C.W., \& Miller, R.R. (2012). Animal models of psychopathology: 
Historical models and the pavlovian contribution. Terapia Psicológica, 30, 45-59.

Legge, E.L.G., \& Spetch, M.L. (2009). The differential outcomes effect (DOE) in spatial localization: An investigation with adults. Learning and Motivation, 40, 313-328.

Lerman, D. C. (2003). From the laboratory to community application: translational research in behavior analysis. Journal of Applied Behavior Analysis, 36, 415-419.

Leslie, J. (2011). Animal models of psychiatric disorders: behavior analysis perspectives. European Journal of Behavior Analysis, 12, 27-40.

Litt, M.D. \& Schreibman, L. (1981). Stimulus-specific reinforcement in acquisition of receptive labels by autistic children. Analysis and Intervention in Developmental Disabilities, 1, 171-186.

López-Crespo, G., Plaza, V., Fuentes, L.J., \& Estevez, A.F. (2009). Improvement of age-related memory deficits by differential outcomes. International Psychogeriatrics, 21, 503-510.

Mace, F.C., \& Critchfield, T.S. (2010). Translational research in behavior analysis: historical traditions and imperative for the future. Journal of the Experimental Analysis of Behavior, 93, 293-312.

Maki, P., Overmier, J.B., Delos, S., \& Gutman, A.J. (1995). Expectancies as factors influencing conditional discrimination performance of children. The Psychological Record, 45, 45-71.

Malanga, P., \& Poling, A. (1992). Letter recognition by adults with mental handicaps: through differential outcomes. Developmental Disabilities Bulletin, 20, 39-48.

Martella, D., Plaza, V., Estévez, A.F., Castillo, A. \& Fuentes, L.J. (2012). Minimizing sleep deprivation effects in healthy adults by differential outcomes. Acta Psychologica, 139, 391-396.

Martínez, L., Estevez, A.F., Fuentes, L.J., \& Overmier, J.B. (2009). Improving conditional discrimination learning in five-year-old children: DOE using different types of reinforcement. The Quarterly Journal of Experimental Psychology, 62, 1617-1630.

Martínez, L., Marí-Beffa, P., Roldán-Tapia, D., RamosLizana, J., Fuentes, L.J., \& Estévez, A.F. (2012). Training with differential outcomes enhances discriminative learning visuospatial recognition memory in children born prematurely. Research in Developmental Disabilities, 33, 76-84.

Mateos, L.R., Cabrera, R., García-Leal, O., \& Flores C. (2011). Evaluación del efecto de resistencia al cambio en un procedimiento de consecuencias diferenciales. Revista Mexicana de Investigación en Psicología, 3, 36-43.

Mateos, L.R., Madrigal, K., Flores, C., \& Overmier, J.B. (2016). The effects of differential outcomes on learning and memory in young and aged rats. Learning and Motivation, 53, 1-6.

Miller, O., Waugh, K, \& Chambers, K. (2002). Differential outcomes effect: Increased accuracy in adults learning Kanji with stimulus specific rewards. Psychological Record, 52, 315-324.

Minster, S.T., Jones, M., Ellife, D., \& Mathukumaraswamy, S.D. (2006). Stimulus equivalence: Testing Sidman's (2000) theory. Journal of the Experimental Analysis of Behavior, 85, 371-391.

Mok, L.W., Estevez, A., \& Overmier, J.B. (2010). Unique outcome expectations as a training and pedagogical tool. The Psychological Record, 60, 227-248.

Mok, L.W., \& Overmier, J.B. (2007). The differential outcomes effect in normal human adults using a concurrent-task within-subject design and sensory outcomes. The Psychological Record, 57, 187-200.

Mustaca, A.E (2004). Tratamientos psicológicos eficaces y ciencia básica. Revista Latinoamericana de Psicología, 36, 11-20.

Mustaca, A.E. (2011). Evaluación objetiva de los tratamientos psicológicos: modelos basados en ciencia. Revista Colombiana de Psicología, 20, 99-106.

Overmier, J.B. (2001). Del laboratorio a la clínica: una parabola moderna. Revista Mexicana de Psicología, $18,287-300$.

Overmier, J.B. (2007). La investigación básica con animales fortalece la ciencia y práctica de la psicología. Interdisciplinaria, 24, 211-228.

Peterson, G.B., Wheeler, R.L., \& Armstrong, G.D. (1978). Expectancies as mediators in the differential - reward conditional discrimination performance of pigeons. Animal Learning and Behavior, 6, 279-285.

Peterson, G.B., Wheeler, R.L., \& Trapold, M.A. (1980). Enhancement of pigeons conditional discrimination performance by expectancies of reinforcement 
and non reinforcement. Animal Learning and Behavior, 8, 22-30.

Petticrew, M., \& Roberts, H. (2006). Systematic reviews in the social sciences, a practical guide. Malden, MA: Blackwell Publishing.

Plaza, V., Antúnez, C., Estévez, A.F., López-Crespo, G., \& Fuentes, L.J. (2012). Improving Delayed Face Recognition in Alzheimer`s Disease by Differential Outcomes. Neuropsychology, 4, 482-489.

Plaza, V., Estévez, A.F., López-Crespo, G., \& Fuentes, L.J. (2011). Enhancing recognition memory in adults through differential outcomes. Acta Psychologica, 136, 129-136.

Plaza, V., Esteban, Estévez, A.F., \& Fuentes, (2013). El procedimiento de consecuencias diferenciales mejora el reconocimiento de rostros con independencia de su valencia emocional. Psicológica, 34, 79-95.

Ribes, E. (2009). Reflexiones sobre la aplicación del conocimiento psicológico: iqué aplicar o cómo aplicar? Revista Mexicana de Análisis de la Conducta, 35, 3-17.

Santoyo, C. (2012). Investigación traslacional: Una misión prospectiva para la ciencia del desarrollo y la ciencia del comportamiento. Revista Mexicana de Investigación en Psicología, 4, 84-110.

Saunders, R., \& Sailor, W. (1979). A comparison of three strategies of reinforcement on two-choice learning problems with severely retarded children. AAESPH Review, 4, 323-333.

Savage, L.M. \& Langlais, P.J. (1995). Differential outcomes attenuate spatial memory impairments on matching to position following pyrithiamine-induced thiamine deficiency in rats. Psychobiology, 23, 153-160.

Savage, L.M., Pitkin, S.R., \& Careri, J.M. (1999). Memory enhancement in aged rats: the differential outcomes effect. Developmental Psychobiology, 35, 318-327.

Savage, L.M., Stanchfield, M.A., \& Overmier, J.B. (1994). The effects of scopolamine, diazepam, and lorazepam on working memory in pigeons: an analysis of reinforcement procedures and sample problem type. Pharmacology, Biochemistry, and Behavior, 48, 183-191.

Tashiro, T., \& Mortensen, L. (2006). Translational research. How social psychology can improve psychotherapy. American Psychologist, 61, 959-966.

Trapold, M.A. (1970). Are expectancies based upon different positive reinforcing events discriminably different? Learning and Motivation, 1, 129-140.

Trapold, M.A., \& Overmier, J.B. (1972). The secondary learning process in instrumental learning. In A. H. Black \& W.F. Prokasy (Eds.), Classical conditioning: Vol 2. Current Research and Theory. New York: Appleton.

Urcuioli, P.J. (2005). Behavioral and associative effects of differential outcomes in discrimination learning. Learning and Behavior, 33, 1-21.

Urrútia, G., \& Bonfill, X. (2010). Declaración PRISMA: una propuesta para mejorar la publicaciuón de revisiones sistemáticas y metaanálisis. Medicina Clínica, 135, 507-511. 